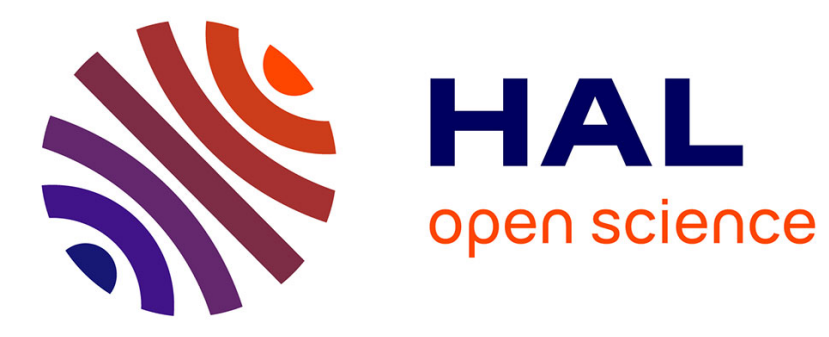

\title{
La sécurité au travail accaparée par les directions
}

Nicolas Jounin

\section{To cite this version:}

Nicolas Jounin. La sécurité au travail accaparée par les directions. Actes de la Recherche en Sciences Sociales, 2006, 165 (5), 10.3917/arss.165.0072 . hal-01822285

\section{HAL Id: hal-01822285 \\ https://hal.science/hal-01822285}

Submitted on 16 Aug 2018

HAL is a multi-disciplinary open access archive for the deposit and dissemination of scientific research documents, whether they are published or not. The documents may come from teaching and research institutions in France or abroad, or from public or private research centers.
L'archive ouverte pluridisciplinaire HAL, est destinée au dépôt et à la diffusion de documents scientifiques de niveau recherche, publiés ou non, émanant des établissements d'enseignement et de recherche français ou étrangers, des laboratoires publics ou privés. 


\section{LA SÉCURITÉ AU TRAVAIL ACCAPARÉE PAR LES DIRECTIONS Quand les ouvriers du bâtiment affrontent clandestinement le danger Nicolas Jounin}

Le Seuil | «Actes de la recherche en sciences sociales »

2006/5 n 165 | pages 72 à 91

ISSN 0335-5322

ISBN 2020908498

Article disponible en ligne à l'adresse :

https://www.cairn.info/revue-actes-de-la-recherche-en-sciencessociales-2006-5-page-72.htm

\section{Pour citer cet article :}

Nicolas Jounin, « La sécurité au travail accaparée par les directions. Quand les ouvriers du bâtiment affrontent clandestinement le danger », Actes de la recherche en sciences sociales 2006/5 ( $\left.\mathrm{n}^{\circ} 165\right)$, p. 72-91.

DOI 10.3917/arss.165.0072

Distribution électronique Cairn.info pour Le Seuil.

(C) Le Seuil. Tous droits réservés pour tous pays.

La reproduction ou représentation de cet article, notamment par photocopie, n'est autorisée que dans les limites des conditions générales d'utilisation du site ou, le cas échéant, des conditions générales de la licence souscrite par votre établissement. Toute autre reproduction ou représentation, en tout ou partie, sous quelque forme et de quelque manière que ce soit, est interdite sauf accord préalable et écrit de l'éditeur, en dehors des cas prévus par la législation en vigueur en France. Il est précisé que son stockage dans une base de données est également interdit. 


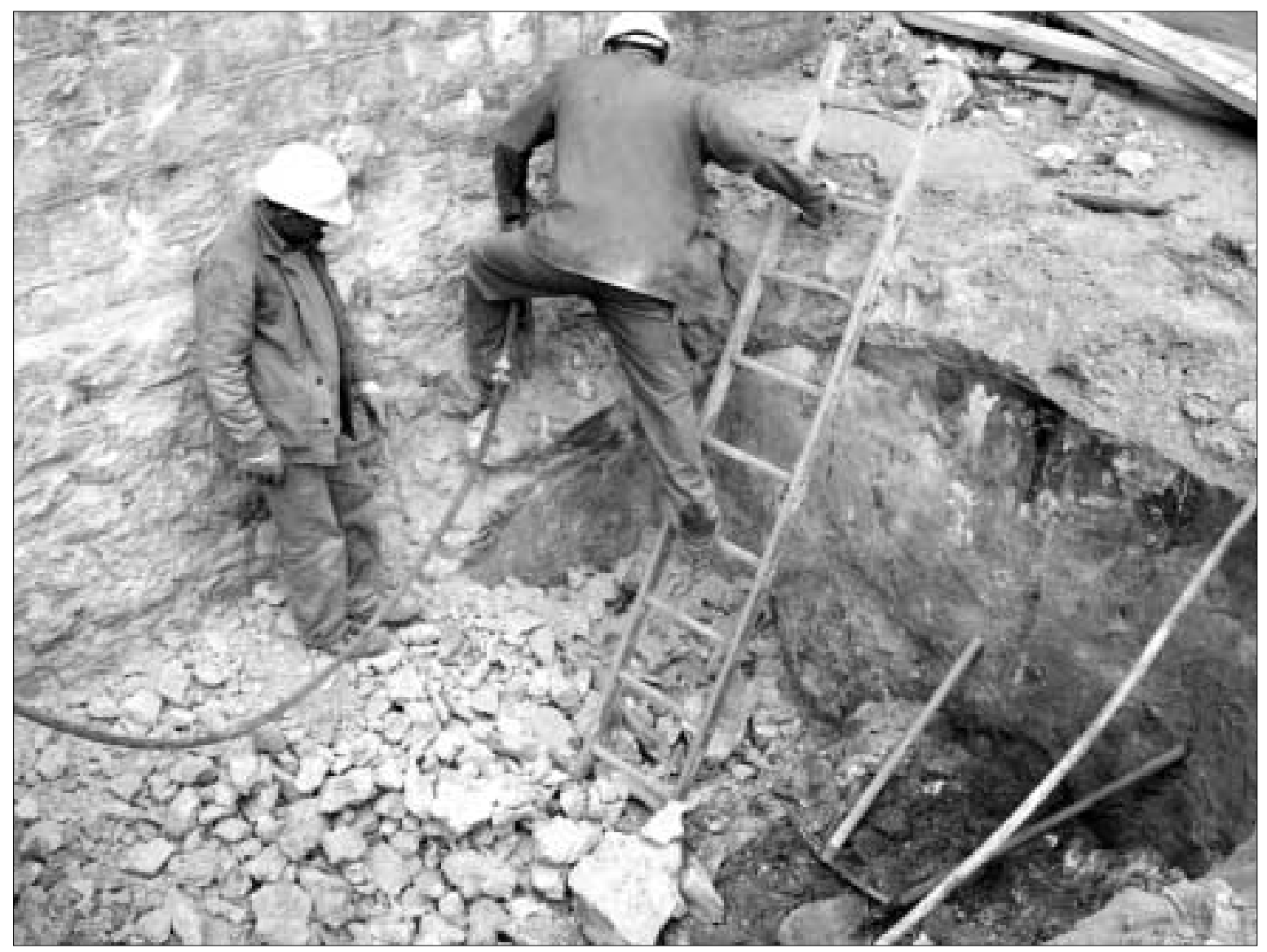

«TU VAS PAS ME DIRE que t'as jamais travaillé sur une échelle. » 


\title{
La sécurité au travail accaparée par les directions
}

\author{
Quand les ouvriers du bâtiment affrontent clandestinement le danger
}

En 2000, le BTP représentait officiellement $21 \%$ des 48000 accidents du travail ayant entraîné une incapacité permanente dénombrés en France, et $26 \%$ des 730 accidents ayant causé un décès, al ors que les salariés du BTP ne représentaient que $7 \%$ de l'ensemble des effectifs salariés. La même année, on compta dans le BTP un accident du travail avec arrêt pour 10 salariés; et un accident du travail ayant entraîné une incapacité permanente pour 120 salariés $^{1}$. II faut se souvenir de ces chiffres, mais cet article n'a pas vocation à démontrer la dureté des conditions de travail ou à faire l'inventaire des risques que rencontrent les ouvriers du bâtiment. Pourquoi, alors, aborder la question de la sécurité? Parce que son étude ne peut se cantonner à une mesure; parce que l'insécurité n'est pas une donnée brute, irréductible à toute emprise humaine, mais qu'elle fait l'objet d'une prise en charge, d'une gestion à travers laquelle se définissent des statuts et des hiérarchies. Au sein de la relation salariale, d'une part; et dans le cadre plus particulier de I'organisation du travail en chantier, d'autre part.

On est habitué à identifier la division entre concepteurs et exécutants dans la définition des modes opératoires, du déroulement des tâches, de l'orientation de la production, bref dans tout ce qui touche directement aux droits du capitaliste en tant qu'il est l'organisateur d'un procès visant à produire, pour son compte, de la valeur. Il est plus étonnant de la retrouver à un niveau qui concerne directement et irréductiblement les exécutants: la sécurité Et pourtant, il est possible de parler de taylorisation de la sécurité, dans la mesure où l'organisation de la protection de l'inté grité physique des salariés reproduit le schéma de la division entre concepteurs et exécutants: les concepteurs décident ce qui est bon pour la sécurité des ouvriers; ces derniers l'appliquent (ou du moins sont censés I'appliquer)2.

On essai era de rendre compte de cette organisation, en montrant que derrière l'apparence d'union sacrée des directions et des ouvriers contre les inspections se dissimulent des antagonismes contenus dans le cadre juridico-politique même de la sécurité. Sur le chantier, ces antagonismes se traduisent par un fort recul critique des ouvriers et la nécessité qui se pose à eux de réguler clandestinement leurs écarts visà-vis des règles de sécurité C'est que la gestion légitime de la sécurité a été accaparée par les directions, qui se trouvent porteuses d'une contradiction entre sécurité et cadence. La condamnation au cas par cas des exécutants fautifs, coupables mais pas responsables, ainsi que l'éclatement du collectif de travail qui construit une déresponsabilisation en cascade complètent cette organisation.

Pour pleinement la comprendre, il faut préciser les modes de mise au travail sur les chantiers du gros œuvre, caractérisés par : 1) l'éclatement du collectif de travail lié à la multiplication des employeurs représentés sur un même site. En ne s'intéressant qu'au gros œuvre, on verra que chaque chantier est dirigé par une entreprise générale; l'entreprise générale y emploie des salariés, y utilise des intérimaires recrutés dans trois ou quatre agences différentes, et travaille en collaboration avec une poignée d'entreprises soustraitantes; ces entreprises sous-traitantes emploient 
leur propre personnel, plus des intérimaires dispersés à nouveau entre trois ou quatre agences. On dépasse ainsi la dizaine d'employeurs pour des effectifs qui ne sont pas supérieurs, certaines fois, à cinquante personnes. 2) La hiérarchisation, qui se superpose à l'éclatement, des travailleurs ainsi rassemblés. Les intérimaires représentaient $30 \%$ à $70 \%$ des chantiers observés. 3) La précarité qui touche une frange de ces travailleurs, précarité qui permet un turn-over ajusté aux fluctuations d'activité; mais qui constitue aussi une menace planant sur certains travailleurs qui, de fait, sont stables (intérimaires permanents). 4) L'utilisation massive d'immigrés qui, pour une partie d'entre eux, voient leurs opportunités d'emplois et leurs capacités de résistance limitées, directement ou indirectement, par les statuts que l'État leur octroie (ou le refus d'octroi d'un statut dans le cas des sans-papiers).

\section{L'union sacrée contre les inspecteurs: la sécurité en représentation}

La sécurité fait l'objet d'une mise en scène quotidienne de la part des entreprises. Par exemple, ce panneau qu'arborent parfois les chantiers: «Aujourd'hui, énième jour sans accident ». Sur un chantier prestigieux où j'ai travaillé, le panneau annonçait, lorsque je suis arrivé: «146e jour sans accident » (pour 146 jours de travail). Pourtant, j'allais apprendre du gardien de chantier que, quelques mois auparavant, un ouvrier était envoyé à I'hôpital (pour presque un an), ayant reçu une charge de ferraille dans le dos (la charge était portée par la grue, et le grutier manœuvrait sans visi bilité, gêné par la façade conservée de ce vieil immeuble haussmannien). Selon le gardien, c'est le directeur du chantier qui a interdit de modifier le panneau sur la sécurité. Quelques semaines après mon départ du chantier, un chef d'équipe intérimaire se blessait à la jambe. Le panneau disparut alors, accroché dans un coin reculé d'Algeco, et le blanc indiquant le nombre de jours sans accident n'était plus renseigné. Quand je signalai ce fait à un conducteur de travaux en entretien, il répondit de manière un peu gênée: «Ouais, mais il y avait un moment qu'il avait pas été mis à jour, ce panneau »; un autre conducteur de travaux qui entendait la conversation rit et ajouta: «À partir du moment où il y a un accident, on le met plus à jour ».

Cette mise en scène de l'irréprochabilité est réactivée à chaque passage d'une inspection de sécurité. Ces visites occasionnent sur les chantiers des scènes parfois proches de la panique (et réduisant, le temps de quelques heures, la productivité du chantier) qui révèlent a contrario les transgressions routinières des règles de sécurité (aussi bien dans les consignes que les cadres distribuent que dans la manière dont les ouvriers les exécutent). Cadres et ouvriers ne donnent à voir aux directions de chantier, coordinateurs sécurité, contrôleurs de l'Organisme professionnel de pré vention du bâtiment et des travaux publics (OPPBTP) ou inspecteurs du travail, bref à tous ceux qui viennent ponctuel lement sur les chantiers en étant habilités à faire des jugements sur la sécurité, qu'une parodie de travail de chantier (même si la parodie ne peut éradiquer miraculeusement toute trace de réalité) ${ }^{3}$.

\begin{abstract}
Cette «parodie» peut être illustrée par une réunion de sécurité d'un chantier de quatre grues, à laquelle je participe en tant que ferrailleur intérimaire. Le ferraillage est sous-traitéà l'entreprise FF. J'ai été désigné quelques minutes auparavant par le chef de chantier de FF pour assister à cette réunion (organisée tous les trois mois, par obligation légale) : «Tu dis oui oui, si on te demande, tu dis que tu parles pas français», rigole le chef, avant de me redire plus sérieusement: «Tu dis que oui tout va bien, même si ça va pas, tu dis oui ». Je suis accompagné par le conducteur de travaux de FF, M. Renoir. Pendant que nous allons dans les locaux où setient la réunion, $M$. Renoir m'explique qu'elle réunit le coordinateur de sécurité, des représentants de la caisse régionale d'assurance maladie, del'OPPBTP, du maître d'ouvrage, parfois de l'inspection du travail ( Alors eux, il faut espérer que non parce qu'ils sont vraiment casse couilles»), des entreprises présentes sur lechantier et des salariés. «D'habitude, je prends un gars qui parle à peine français, il dit juste [caricaturant un accent arabe] : "Travail tris bien, tris content" ». «IIs vont demander à la fin aux repré sentants de salariés s'ils ont quelque chose à dire sur la sécurité, ou l'hygiène des vestiaires, donc là il faut dire que tout va bien. Parce qu'il y a notre position de sous-traitant, si on dit que quelque chose va mal, c'est contre notre client, et après il va se retourner contre nous. » Pendant que le coordinateur de sécurité dresse une liste, «éloquente» dit-il, des problèmes qu'il a relevés et le nombre de fois où il les a relevés (travail sur une échelle, utilisation de la tronçonneuse sans lunettes, etc.), M. Renoir m'explique ce qu'est le «PPSPS», le plan sécurité que chaque entreprise doit fournir avant d'intervenir sur le chantier: «C'est là que tu dis tout ce que tu comptes faire pour la sécurité, même si généralement tu l'appliques pas. Tu vas pas me dire quet'es jamais montésur uneéchelle pour travailler par exemple».
\end{abstract}

Ce qui frappe dans ce type de scènes, c'est l'union qui prévaut entre ouvriers et cadres dans la tentative de masquer toutes les carences du chantier en matière de sécurité. Certes, les ouvriers prendraient des risques 


\section{Méthodes dienquêtre}

Cet article repose sur un travail de terrain effectué entre 2001 et 2004, dans le cadre d'une recherche de doctorat sur les relations de travail sur les chantiers du bâtiment, et plus précisément du gros œunre. Ce travail de terrain est constitué principalement de:

- une cinquantaine d'entretiens. Quelques-uns exploratoires avec des syndicalistes (syndicats patronaux et de salariés), des institutionnels (ministère de l'Équipement, inspection ou médecine du travail). Et surtout avec des individus agissant à différents niveaux de gestion de la main-d'œure: responsables de ressources humaines des principaux groupes; cadres de chantier (chefs de chantier et conducteurs de travaux); commerciaux d'intérim; et ouvriers du bâtiment;

- douze mois d'obsenvation participante, sous plusieurs formes: 1) six semaines comme manœuvre intérimaire (plusieurs missions, dont une de quatre semaines), en 2002 (obsenvateur inconnu). 2) Quatre mois et demi par le biais d'une formation en coffrage et ferraillage (connu), comprenant un stage de coffreur de six semaines sur un chantier de gros œuvre (inconnu), en 2003. 3) Trois mois comme stagiaire ferrailleur dans une entreprise sous-traitante en ferraillage, sur trois chantiers, en 2004 (connu). 4) Toujours en 2004, trois mois comme intérimaire (inconnu), d'abord six semaines comme aide-coffreur (entreprise générale), puis quelques courtes missions comme manœumre, et quatre semaines comme ferrailleur (entreprise sous-traitante).

\section{La distinction entre obsenvateur connu} et inconnu signale le fait que les directions de chantier ou d'entreprise étaient, ou non, au courant de mon enquête. Dans les cas où j'étais inconnu, je me «dévoilais » à mes collègues de travail dès lors que suffisamment de confiance était établie. Outre des obsenvations sur l'organisation du travail au quotidien et les relations pratiques et verbales entre acteurs des chantiers, l'obsenvation participante a permis d'accumuler des conversations informelles venant s'ajouter aux entretiens enregistrés.

Les notes de terrain ont été prises quotidiennement. 
s'ils remettaient en cause ouvertement ces mises en scène, et ils les critiquent lorsqu'ils se retrouvent à part. Mais pour eux, les contrôleurs ne sont pas des sauveurs. Les commentai res des ouvriers à propos des visites, même celles d'inspecteurs extérieurs et indé pendants, sont généralement désobligeants.

\begin{abstract}
Sur un autre chantier, Tonio, un coffreur qualifié, prend sur lui de placer régulièrement des gardecorps, malgré les consignes d'un chef d'équipe qui l'envoie sur une autre tâche. Tonio persiste: «lls s'en fichent de la sécurité. Et quand la sécurité passe, il y a tout le monde qui court ». C'est bien anticipé de sa part, et il s'en félicitera, car l'aprèsmidi il y a une visite del'inspectrice del'OPPBTP: «Je le savais, je me disais que ça faisait longtemps qu'elle était pas venue», dit Tonio à plusieurs chefs d'équipe en racontant qu'il a placé les gardecorps. Escalier trop raide, ferrailles qui traînent, entre autres choses: Tonio suppose qu'un avertissement va tomber. II dit pourtant de l'inspectrice que c'est une «conne», qui «connaît rien». «Reprocher à quelqu'un de mal avoir fait son boulot, d'accord, si on sait mieux le faire que lui. Elle est là juste pour dire que ça va pas. Elle est payée pour ça. »
\end{abstract}

II serait facile de conclure que les ouvriers sont leurrés par l'idéol ogie du «tous dans le même bateau », ou qu'ils ont intériorisé, avec les contraintes de cadence, une attitude d'hostilité aux inspecteurs qui pourtant seraient là pour les protéger. En fait, on perçoit aussi, dans ce dernier exemple, l'hostilité des ouvriers envers la maîtrise. On ne peut comprendre ce double ressentiment, souvent voilé et clandestin, sans revenir à l'encadrement juridique de la sécurité (qui renvoie plus largement à celui du salariat) et aux rapports deforces qu'il suscite.

\section{Patrons, salariés et État: la construction politique de la sécurité}

Hors syndicats de travailleurs, les organisations du secteur du BTP, professionnelles ou institutionnelles, se cantonnent publiquement à une approche technique de la sécurité Ainsi l'OPPBTP (organisme paritaire) instaure-t-il des «baromètres » de la sécurité, des «analyses des mesures de prévention technique», etc. L'analyse de l'insécurité au travail se limite al ors à sa mesure; symétriquement, la lutte engagée contre elle prend la forme de prescriptions, d'incitations, de «campagnes de sensibilisation», de «diffusion de bonnes pratiques», et autres formes habituel les de la «pédagogie» institutionnelle, relayée par les entre prises. L'insécurité au travail n'est vue que comme une somme de mauvaises habitudes à combattre l'une après l'autre, tout en rappelant que les effets de ce combat sont nécessairement longs à venir, et que «le risque zéro n'existe pas», comme l'exprime ce conducteur de travaux:

\begin{abstract}
«Au final, il y a eu plein de choses qui sont devenues des habitudes, foutre un garde-corps, vérifier lorsquet'élingues à la grue quec'est bien élingué, plein de choses qui sont des habitudes. Porter le casque, c'est rentré, ça a mis du temps à rentrer... Les casques et les bottes de sécurité, ça a mis du temps à rentrer dans les mœurs. Maintenant c'est rentré. II faut que tout devienne une habitude $[\ldots]$ II faut toujours vérifier, toujours être là, toujours leur redire. [...] Et c'est pas des choses qui se changent en une semaine, en un mois ou en un an. [...] On essaie de le diminuer, le diminuer, le diminuer, notre objectif c'est toujours zéro accident, mais... c'est une limite presque inatteignable C'est mathématique. Quand tu bricoles chez toi... Sur toute ta vie pendant 30 ans, tu vas bricoler un petit peu, tu vas te péter le doigt une fois, voilà. Tu vas te taper sur le doigt une fois. Sauf que sur un chantier, il y a plus d'heures, parce que tu fais ça toute la journée, donc tu vas te taper sur le doigt une fois par semaine. En probabilité. »
\end{abstract}

On trouve là un condensé des visions patronale et institutionnelle sur la sécurité, caractérisées par une série de réductions: 1) techniciste: la question de la sécurité n'est que celle de la recherche et de l'application des mesures adéquates, le risque provenant donc exclusivement de «l'erreur humaine» des opérateurs $\left.{ }^{4} ; 2\right)$ évolutionniste: les mentalités changent inéluctablement sous l'effet de la pédagogie (I'évolutionnisme autorisant à pester, a contrario, contre le «retard des mentalités») ; 3) fataliste: le risque ne sera jamais écarté, puisque toute activité humaine est risquée; 4) découlant de tout le reste, apolitique: la question de la sécurité est ramenée à la condition et à l'activité humaines en général (autorisant une comparaison avec le bricolage), extirpée des cadres, des enjeux et des intérêts antagonistes du travail salarié. II n'est pas question de dire ici que le risque zéro est atteignable; mais que, si le risque physique est inhérent aux activités considérées, il est formal isé et organisé de manière différente selon les rapports sociaux qui encadrent ces activités. La vision à sens unique de «mentalités à changer » élude le fait que la prise en charge de la sécurité, en dépit de tous les discours affirmant que c'est «l'affaire de tous », est dans la pratique l'enjeu d'un pouvoir inégalement réparti. 
Pourtant, le débat virulent autour de la loi de 1898 sur les accidents du travail montrait son importance politique. II s'agissait de répondre à cette question, portée par le mouvement ouvrier : sur quel principe fonder la réparation des accidents du travail, et contraindre les patrons à participer à cette réparation? En inventant la théorie du risque professionnel, la loi instituait une responsabilité patronale automatique mais limitée : le patron est responsable del'accident du travail non parce qu'il a commis une faute (non-respect de règles de sécurité, fourniture de matériel défectueux, etc.) mais parce qu'il place ses salariés en situation de danger du fait même qu'il dirige leur activité5. C'est la subordination du salarié qui fonde la responsabilité de l'employeur. Cela écarte toute comparaison valable entre bricolage domestique et travail salarié, puisqu'il est reconnu que la victime de l'accident n'est pas l'instigatrice de l'activité qui a provoquél'accident. C'est en tant que subordonné à une autre volonté, réputée supérieure par contrat, que letravailleur a agi ; c'est sur ordre En instituant une nouvel le responsabilité dans I'accident, qui n'était plus seulement cel le du travailleur accidenté, la loi de 1898 fondait du même coup le droit du travail comme droit spécifique détaché du droit contractuel : droit de la subordination consentie par contrat. «C'est parce que dans l'exécution de ce contrat le corps du salarié cesse d'être le siège d'une libre volonté individuelle, pour devenir une chose vivante insérée dans une organisation conçue par autrui, que la personne du sala rié cesse d'être tenue pour responsable de ce qu'il advient de son propre corps », écrit Alain Supiot ${ }^{6}$.

Aussi les contradictions qui fondent la reconnaissance légale du salariat rejaillissent-elles sur la sécurité. Contradictions que l'on peut résumer ainsi : reconnaître le sal ariat, lefonder sur le fait de la subordination, c'est permettre d'édifier des protections en faveur de ces subordonnés; mais c'est aussi entériner qu'on puisse aliéner sa volonté individuelle dans le cadre d'un contrat, et que l'État soit le passage obligé d'une limitation du pouvoir patronal. C'est parce que les salariés se dépossèdent temporairement de leur volonté pour devenir l'instrument des plans du capitaliste, c'est parce qu'ils se privent, contractuellement, de la possi bilité de se prendre en charge et de décider de leurs mouvements, qu'il ne reste plus que la loi pour restreindre le pouvoir de l'employeur, notamment en matière de sécurité Cette restriction de pouvoir sefait au coup par coup, point par point, lutte après lutte, cas concret après cas concret. Et elle se traduit par un cortège de réglementations qui rend incantatoire la formule selon laquelle «nul ne doit ignorer la loi ». Le volumineux Code d'hygiène et de sécurité est I'hé ritier de la loi de 1898. La dépossession des salariés de l'organisation de leur propre sécurité fait de celle ci un compromis permanent entre les vues des capitalistes et les règles étatiques, ces dernières établissant pêle-mêle la hauteur minimale des garde-corps, le poids autorisé sur les monte-charges, la fréquence des réunions de sécurité, l'inventaire des protections individuelles, etc. ${ }^{7}$. La professionnalisation du contrôle de la sécurité engendre une logique plus policière que pré ventive: «Les agents du contrôle observent et verbalisent plus volontiers sur ce qui est techniquement, juridiquement plus facile à établir [...] que sur ce qui occasionne le plus d'accidents ${ }^{8}$ ». Ou plutôt ce contrôle ne peut-il faire autrement que d'osciller entre une logique de sanction systématique et une logique «compréhensive» et conciliante à l'égard des directions de chantier. Car, que l'État punisse les employeurs ou négocie avec eux les conditions du travail, les salariés ne sont jamais, pour les politiques de sécurité, que des sujets: aux sens de matière principale et d'assujettis.

Mais la loi n'oublie pas complètement que le salariéest capable d'agir, et d'agir mal. À côté d'arrêts qui renforçaient la possibilité d'invoquer la «faute inexcusable» del'employeur'9, la Cour de cassation rend le 28 février 2002 un arrêt étendant de même le champ de la faute éventuelle du salarié Selon le Code du travail, il incombe à ce dernier «de prendre soin, en fonction de sa formation et selon ses possibilités, de sa sécurité et de sa santé ainsi que de celles des autres personnes concernées du fait de ses actes ou de ses omissions au travail », et ce «conformément aux instructions qui lui sont données par l'employeur ou le chef d'établissement » (article L. 230-3 du Code du travail). Formule ambiguë, qui laisse planer le doute sur la nature de la faute susceptible d'être reprochée au salarié. Est-ce de ne pas prendre soin de la sécurité, ou de ne pas respecter les consignes de sécurité posées par l'employeur? Dans son arrêt, la Cour justifie un licenciement pour faute grave en vertu de cette obligation du salarié de veiller à la sécurité, mais sans référence à l'exigence de conformité aux instructions de l'employeur ${ }^{10}$. L'obligation de veiller à la sécurité pesant sur le salarié prend ainsi une portée générale, et rapproche singulièrement les obligations du travailleur de celles de son employeur. Cequi poseau moins deux problèmes: 1) Présentée comme une obligation découlant du
5. J eanf acques Dupeyroux, «Un deal en béton?», Droit social, 7-8, juillet-août 1998, p. $631-634$.

6. Alain Supiot, Critique du droit du travail, Paris, PUF, 2002 (1994), p. 70
7. Annie Thébaud-Mony (op. cit., p. 12) écrit ainsi : «La construction de la "paix sociale" entre le patronat et le mouvement syndical, autour des questions de santé et de sécurité, s'est faite à travers le triomphe de l'approche technique de l'accident et de sa prévention

8. P. Trinquet, op. cit., p. 119

9. Matthieu Babin et Nathalie Pichon «Obligation de sécurité et faute inexcusable de l'employeur », Droit social, 9-10, septembre-octobre 2002, p. 828-839. 10. Alain Couret, «La responsabilité du salarié en matière de sécurité et de préven tion des risques professionnels », Rapport annuel de la Cour de cassation, 2002. 


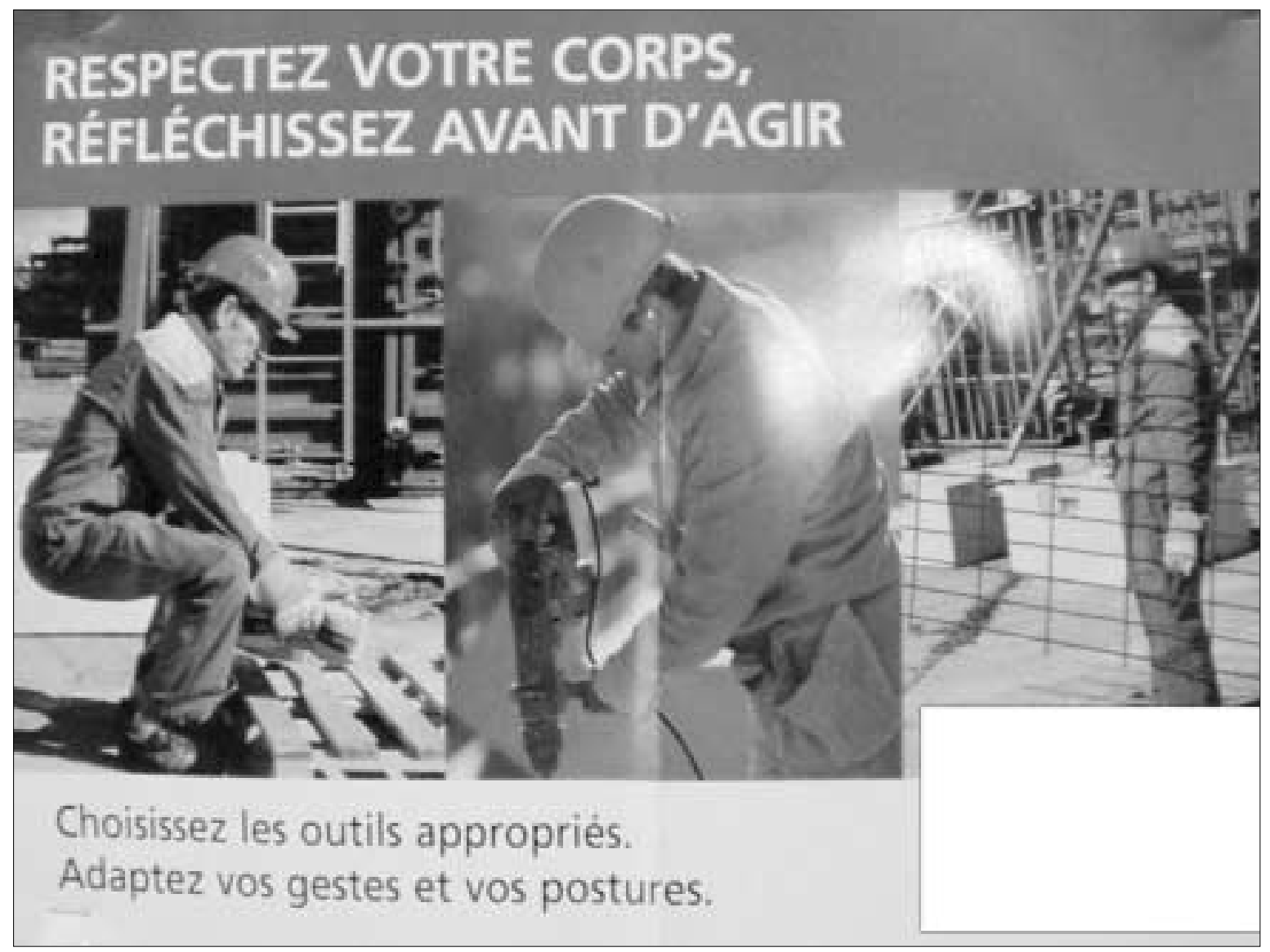

UNE AFFICHE d'entreprise concernant la sécurité. 


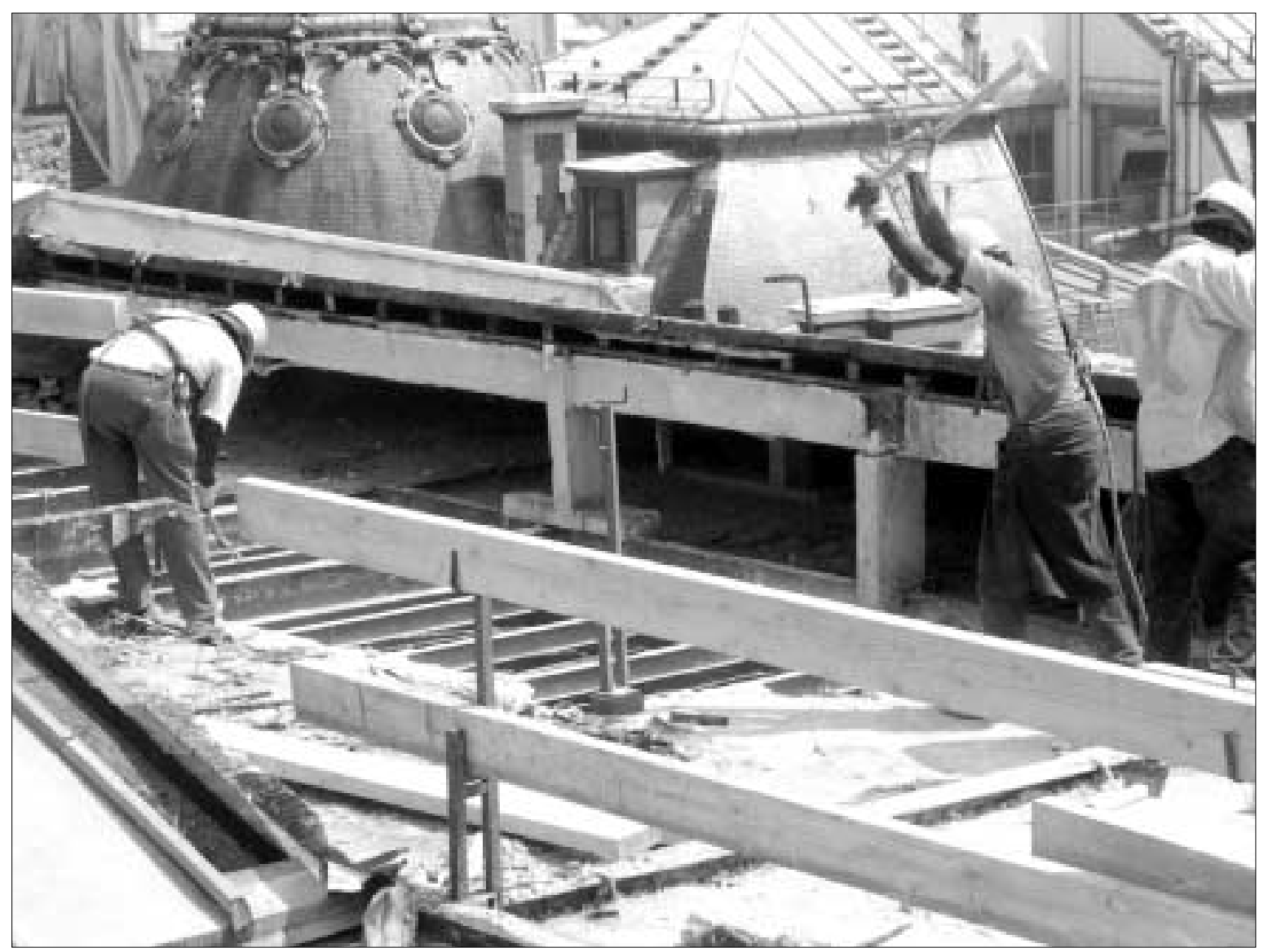

LA DÉMOLTION est la partie la plus dangereuse d'un chantier. 
contrat de travail, qu'en est-il si cette obligation de sécurité entre en contradiction, dans la pratique, avec cette autre obligation contractuelle qui est de se soumettre à l'employeur? 2) Le cadre du contrat de travail demeurant celui de la subordination, le respect ou non de cette obligation de sécurité par le salarié peut être soumis au jugement et au contrôle disciplinaire de l'employeur. Autrement dit: celui qui place les travailleurs en situation de danger se trouve être le même qui les juge fondés à écarter ou non ce danger.

Une pratique patronale vient confirmer cette contradiction: c'est celle qui institue des primes pour les travailleurs en fonction de la fréquence des accidents constatés dans l'équipe de travail. Ces primes sont courantes dans le BTP, et c'est d'ailleurs à partir du cas d'une entreprise de BTP, la SCREG (groupe Bouygues), que la Cour de cassation a eu à se prononcer sur la légalité de ces primes le 24 septembre 200211. La Cour validait l'institution de cette prime en recourant, à nouveau, à l'article L. 230-3 du Code du travail (obligation de sécurité imposée au salarié). Il est probable que l'institution de telles primes fasse baisser le nombre des accidents déclarés, et de ce fait les coûts de l'entreprise; mais on ne peut dire, sans enquête complémentaire, si c'est parce que les salariés font davantage attention ou si c'est parce qu'ils masquent les lésions que leur occasionne le travail. On en arrive ainsi à une situation où les bénéficiaires théoriques du système de protection peuvent en venir à cacher les accidents dont ils sont victimes ${ }^{12}$.

Aussi l'obligation de veiller à la sécurité qui pèse sur le salarié n'est-elle pas véritablement une entorse au principe de subordination : el le limite la responsabilité de l'employeur tout en étendant son pouvoir, celui de juger du comportement des salariés vis-à-vis de la sécurité. La responsabilité qui pèse sur les salariés n'est pas de l'ordre de la prise en charge autonome de la sécurité; elleest enfermée dans la relation de subordination. En fait, un des seuls cas où les salariés sont juridiquement appelés à organiser la sécurité par eux-mêmes et pour eux-mêmes ${ }^{13}$, où ils sont fondés à contester le principe de subordination, tient dans le droit de retrait d'une situation dangereuse, octroyé par le législateur en 1982 (article L. 231-8-1 du Code du travail). Dans ce cas, la protection des travailleurs repose sur leur propre conscience et non sur des réglementations étatiques (même si, là encore, le recours à
l'État s'est révélé nécessaire dans la définition et la restriction de ce droit: les jurisprudences se sont multipliées, qui se chargent de contrôler le «motif raisonnable de penser [que la situation de travail] présentait un danger grave et imminent »- qui seul fondele droit des salariés à se retirer).

Pourtant, à l'encontre de ce que laissait imaginer ce nouveau droit (des salariés contestant ouvertement les situations dangereuses auxquelles ils sont confrontés), on observe tout autre chose sur les chantiers du gros œuvre: non seulement des ouvriers prennent des risques pour tenir les cadences au sein del'organisation du travail qui leur est imposée, mais ils cachent aux cadres qu'ils prennent des risques, par peur de sanctions.

\section{Du côté des ounriers : prendre des risques et cacher qu'on prend des risques}

\begin{abstract}
Sur un chantier de grosse réhabilitation (seules les façades sont conservées), une opération particulièrement délicate consiste à placer des poutres en béton, préfabriquées, descendues par la grue, mais qui doivent être guidées à la main. Chaque poutre va d'un pilier à l'autre. Du béton des poutres sortent une dizaine d' «amorces » (des barres de fer) horizontales; de même des amorces verticales sortent des piliers. Lors de la pose des poutres, les premières doivent s'insérer entre les secondes. Les ouvriers qui dirigent les poutres doivent monter sur des «tours», c'est-à-dire de petits échafaudages sur lesquels on dispose, en haut, des madriers qui vont soutenir les poutres; les madriers servant également de garde-corps pour les ouvriers qui sont à l'intérieur de la tour. Mais les amorces sortant du pilier étant toujours très hautes, il est impossible de guider la poutre descendue par la grue et de faire glisser ses amorces entre celles du pilier en demeurant dans le «panier », c'est-à-dire le plateau de la tour sur lequel on est encore protégé par les garde-corps. II faut donc systématiquement monter sur les madriers, et même parfois sur la poutre encore en suspension, pour pouvoir guider cette dernière correctement; ce qui implique donc de travailler à cinq mètres du sol environ sans aucune protection, en maniant un matériau (la poutre) dont on ne peut maîtriser la force. Une fois où nous sommes plusieurs à être juchés sur les madriers, Tonio me dit soudain: «Redescends, on va être vus! ». Lui aussi redescend vite fait, et avertit les deux coffreurs del'autre pilier qui retournent alors dans le panier. Depuis un étage du bâtiment $B$, je
\end{abstract}

11. Bernard Gaurian, «Obligation du salarié et performances de l'entreprise en matière de sécurité», Droit social, 12, décembre 2002, p. 1054-1058.

12. Les chiffres de 2004 concernant les accidents du travail dans le bâtiment montrent une diminution des accidents déclarés dans leur ensemble, mais une augmentation des accidents graves et du nombre de tués (d'après les Indicateurs sociaux de la Fédération française du bâtiment). Ce qui peut s'interpréter de deux manières: 1) Ces chiffres reflètent des évolutions réelles, ce qui signifierait que les techniques et les manières de les mettre en œuvre occasionnent des accidents moins fréquents mais plus graves. 2) $\mathrm{Si}$ l'on fait l'hypothèse que le nombre global d'accidents évolue dans le même sens que celui des accidents les plus graves, on déduit que les accidents relativement bénins sont parfois masqués - ce qui est à peu près impossible en cas d'accident grave. 13. On ne parle pas ici des comités d'hygiène, de sécurité et des conditions de travail (CHSCT), qui sont des institutions représentatives qui ne peuvent que négocier, en marge des situations concrètes, les règles de sécurité qui s'appliqueront Le fait quíls n'exis tent que dans les entreprises de plus de 50 salariés diminue leur portée dans le bâtiment, en raison de la sous-traitance et de l'intérim 
vois un des conducteurs de travaux nous regarder. Nous attendons qu'il disparaisse pour reprendre la pose de la poutre.

On pourrait multiplier les exemples de ce type. Dans ces cas-là, ce sont indissociablement le matériel et l'organisation du travail qui créent l'insécurité: il faudrait plus de temps, donc modifier l'organisation du travail, pour ajuster le matériel (ajouter des gardecorps expressément pour la pose des poutres, et les enlever ensuite). Ce sont donc des éléments sur lesquels les ouvriers n'ont aucune prise: ni dans l'organisation prescrite ni dans l'organisation réelle du travail. Rien ne peut être amélioré du point de vue de la sécurité dans ces opérations sans la volonté des cadres de chantier. Et pourtant, les ouvriers, face aux cadres, font comme s'ils étaient coupables.

Serait-ce là un cas aigu de mystification, d'intériorisation, les ouvriers percevant les situations avec les yeux et les mots de ceux qui les dominent, et agissant en conséquence? L'arme symbol ique dont se serviraient cadres, directions de chantier et entreprises, pour imposer leur vision de la sécurité sur les chantiers, serait dans ce cas la pédagogie, qui «devient violence symbolique dans la mesure exacte où el le se pré sente comme un rapport de pure communication, et dissimule tout ce qu'elle doit aux rapports de pouvoir et aux inégal ités sociales et culturelles qui en sont le ressort souterrain ${ }^{14} \gg$. Une pédagogie qui se persuade, et persuade, que tout en matière de sécurité est affaire de respect des normes prescrites, où aucune contradiction d'intérêts ne vient troubler la marche du «tous ensemble pour la sécurité». Une pédagogie qui intime, prescrit, décrète, commande et recommande: «Installez des protections efficaces», «Éliminez les causes de chute», «Choisissez les outils appropriés», «Adaptez vos gestes et postures», «Respectez votre corps, réfléchissez avant d'agir », indiquaient ainsi des affiches de l'entreprise sur le chantier dont il était question.

L'idée d'une mystification semble rendre compte de cette situation où les ouvriers sont tellement pris dans la relation de domination qu'ils cachent aux dominants les risques corporels que leur fait prendre cette domination. Mais elle laisse supposer une com plicité des domi nés sans mettre en lumière les efforts des dominants pour contraindre, pour extorquer cette complicité. Elle risque de faire croire que les dominés consentent là où ils ne font que céder ${ }^{15}$, c'est-àdire qu'ils adhèrent véritablement aux raisons que les dominants allèguent pour les soumettre Dans le cas de la sécurité, ce serait croire que les ouvriers repren- nent le discours selon lequel ils ont des mental ités attardées à transformer et des attitudes irresponsables à contrôler. Or, tandis que dans leurs pratiques les ouvriers, par leurs écarts mêmes et le fait qu'ils les cachent, semblent souscrire aux règles des directions, leurs discours contestent généralement l'organisation du travail et les risques que la cadence fait peser sur la sécurité. Ainsi, sur le chantier dont il était question, un tract intersyndical affiché dans le réfectoire dénonçait «toujours plus de travail, toujours plus de cadence, toujours plus d'accidents». Accusation reflétant un discours quotidien des ouvriers, qui dénonce le lien entre les cadences imposées par les directions et l'insécurité subie.

On trouve aussi dans ces discours des critiques ciblées et concrètes des modes de prise en charge de la sécurité par les chefs. Par exemple sur ce même chantier, un coffreur se dit énervé parce qu'un chef n'a pas voulu perdre de temps à mettre le bon crochet à la grue «A près ils pleurent: "Oh, on a des accidents." » Sur un autre chantier, un traceur conteste plus largement la manière dont est gérée la sécurité à travers l'institution des secouristes, dont il fait partie: «[À un stage de secourisme organisé par l'entreprise] ils nous ont dit: "Seul le secouriste décide s'il faut appeler une ambulance ou aller à l'hôpital." J'avais envie de dire: "C'est ça, on l'enterreet on n'en parle plus" ». On retrouvera ce problème avec la question des secouristes (cf. infra), qui est loin d'être unique ment technique.

Si les comportements des ouvriers ne correspondent pas à ce discours, c'est que les directions de chantiers ont réussi à s'instituer, dans les relations qu'elles entretiennent avec les ouvriers (et non dans les consciences mêmes des ouvriers), comme les seules gardiennes légitimes de la sécurité, et à instituer les exécutants comme les seuls responsables des écarts à la sécurité. Ce qui contraindrait les ouvriers à se mettre en représentation, à masquer parfois à leurs supérieurs leurs réelles conditions de travail, imposées par le réel technique, afin d'éviter les réprimandes. Si les inspecteurs sont mal vus des ouvriers, c'est parce qu'ils ne sont pas en mesure de lever cette contrainte; à la limite, ils la renforcent, en commandant aux entreprises d'imposer aux ouvriers un surcroît de travail au nom de la sécurité. Si les ouvriers semblent parfois si «inconscients 》 de ce que les règles de sécurité sont établies «pour leur bien », c'est que ces mêmes règles se trouvent souvent être des armes utilisées à leur encontre, tandis que les moyens de les respecter leur font défaut. 


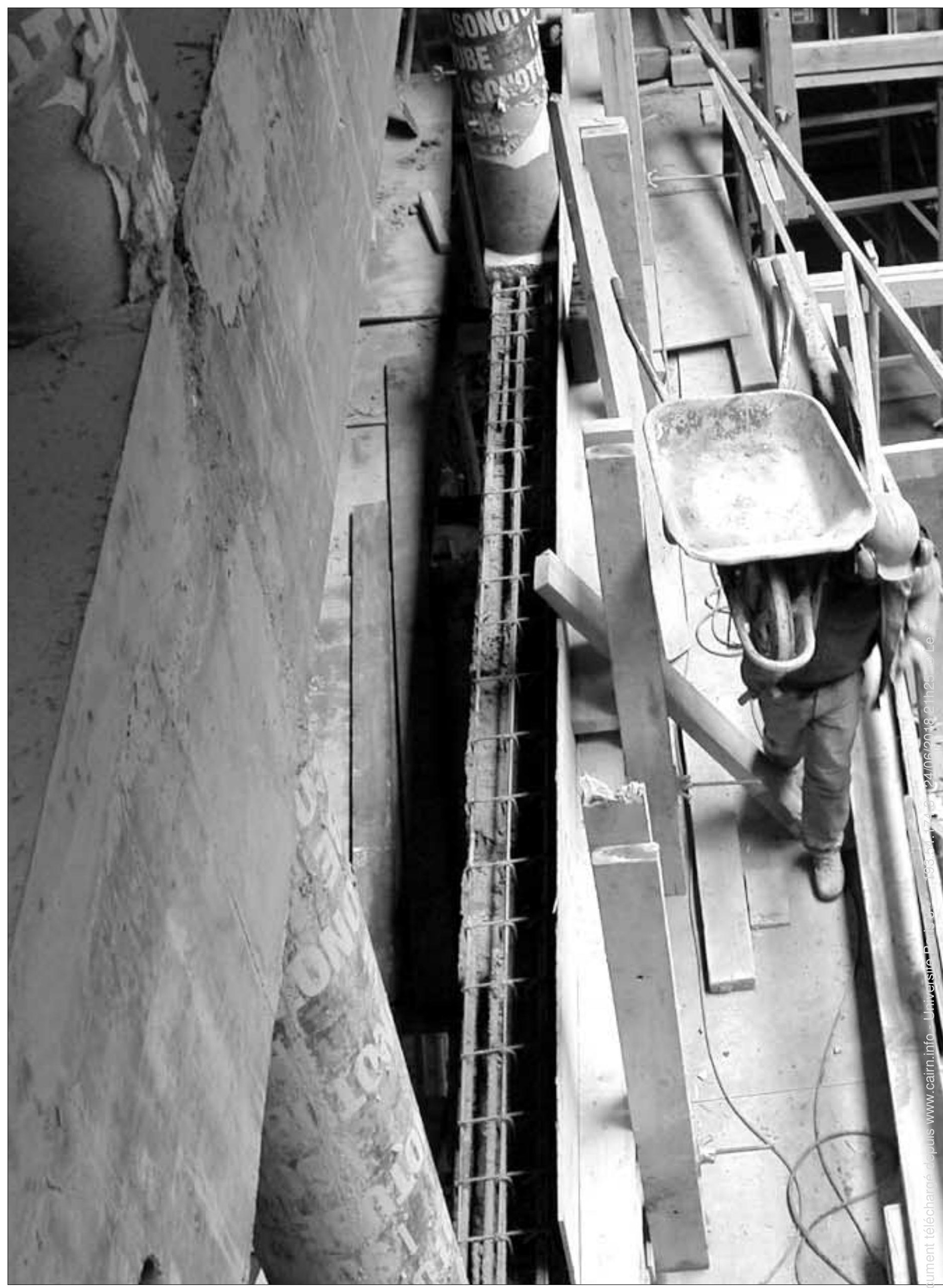




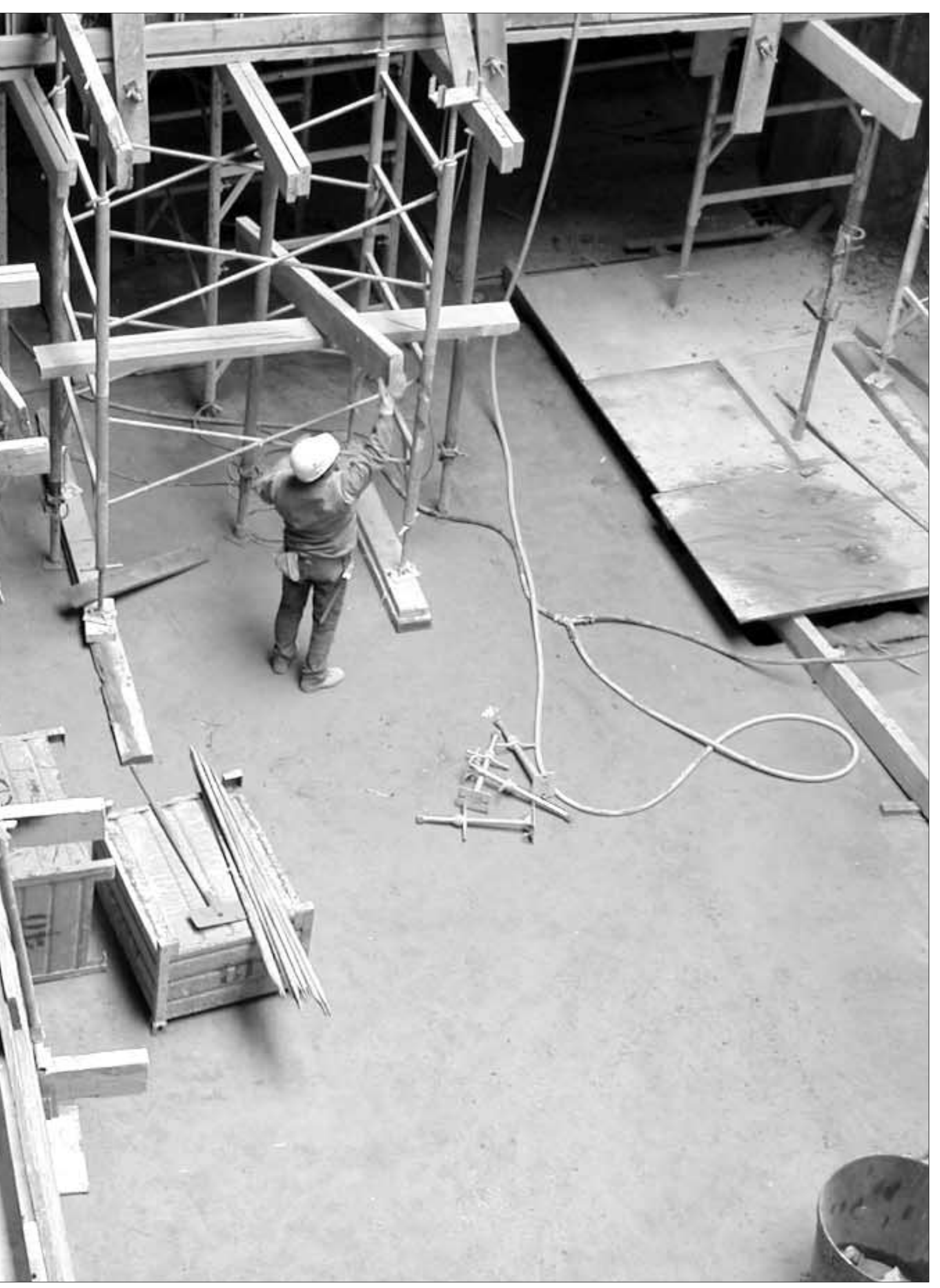




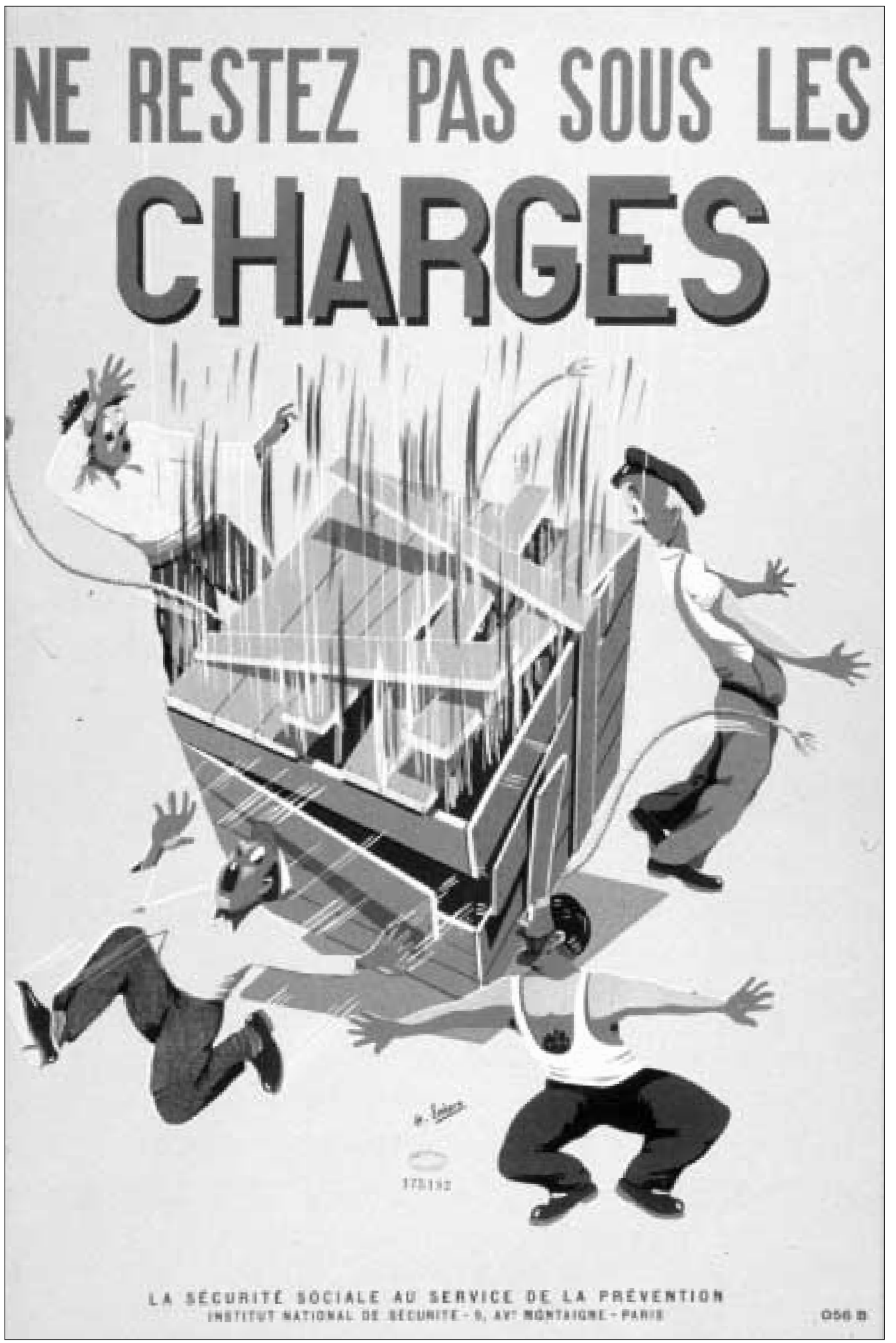

H. EINHORN, vers 1950. Affiche 60 ×40, Bibliothèque Forney, Paris. 


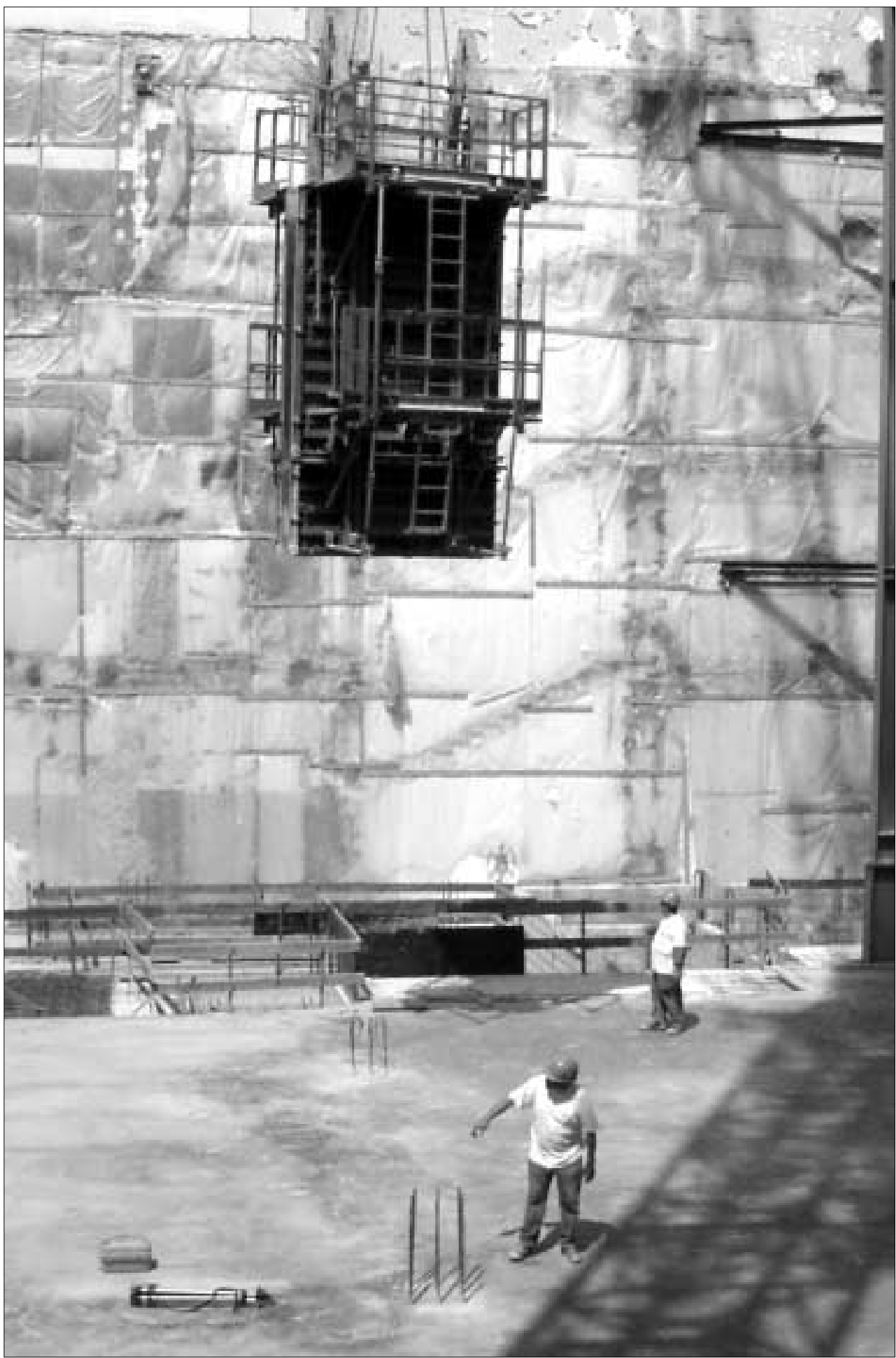

UNE BANCHE portée par la grue au-dessus du chantier. 
Une autre manière de comprendre l'affrontement du danger par les ouvriers, plus dialectique, a été proposée par le psychodynamicien du travail Christophe Dejours, pour une enquête conduite à la fin des années 1970. Il part du constat que «tout se passe commesi les ouvriers du bâtiment étaient inconscients des risques qu'ils encourent, voire comme s'ils y trouvaient un certain plaisir. [...] Les attitudes vis-à-vis du risque d'accident sont bien connues. Le refus de certaines consignes de sécurité aussi ${ }^{16} \gg$. Les ouvriers semblent même parfois aller au-devant du danger, dans des prises de risque inutiles où se jouent des rivalités et des virilités. Mais, dans un contexte où il n'est pas possible de reculer (sauf à perdre son emploi), ces bravades seraient un moyen d'apaiser la peur, d'apprivoiser le danger en le provoquant. «Les ouvriers ajoutent parfois au risque du travail celui de performances personnelles et de véritables concours d'habileté et de bravoure. Dans ces épreuves, ils rivalisent entre eux, mais ce faisant, tout se passe comme si c'était eux qui créaient le risque de toutes pièces et non plus le danger qui s'abattait sur eux indépendamment de leur volonté Créer la situation ou l'aggraver, c'est dans une certaine mesure en être maîtrel7. » C'est ce que Dejours appelle «l'idéologie défensive de métier », dont l'entretien, insiste-t-il, ne peut être assuré que par un collectif. Vingt ans plus tard, j'ai rarement observé ce type de bravades 18 ou de rituels collectifs d'affrontement du danger. Lorsque cela se produisait, il s'agissait d'ouvriers assez âgés ou expérimentés, embauchés ou au statut relativement stable Mais leurs attitudes ne sont pas forcément appréciées, notamment des intérimaires, des sous-traitants, des jeunes, qui raillent les prises de risque inutiles et pestent contre les conditions d'insécurité qu'on leur inflige. Ce constat n'invalide pas les observations de Christophe Dejours, et se comprend à l'intérieur de son cadre d'anaIyse: I'éclatement des collectifs de travail des chantiers explique l'impossibilité de nourrir et cristalliser une idéologie défensive de métier au sein de laquelle les ouvriers puissent se reconnaître et s'investir. Car «il faut, pour la constituer [l'idéologie défensive], la participation d'un groupe ouvrier, c'est-à-dire non seulement une collectivitétravaillant dans un mêmelieu, mais un travail exigeant une répartition des tâches entre les membres d'une équipe ${ }^{19} \gg$.

L'éventual ité d'une «mystification de la conscience» des ouvriers, même sous la forme active et dynami que de «l'idéologie défensive de métier », ne suffit pas à rendre compte de leur attitude. Quelles que soient les médiations psychologiques par lesquelles ils passent, leurs comportements ne peuvent se comprendre que relativement à l'action des dominants, des cadres, et aux dispositifs pratiques de contrainte et de sanction qu'ils mettent en place. La taylorisation de la sécurité, la «prise en charge» de la sécurité par les cadres et la punition des exécutants «fautifs», mais aussi l'éclatement et la hiérarchisation interne du collectif de travail, constituent les dispositifs en question.

\section{Cadence et prudence: des exigences contradictoires portées par une même hiérarchie}

Les directions ont accaparé la sécurité. Non seulement le langage de la sécurité, mais la conception et la gestion des règles elles-mêmes. Au niveau du chantier, ce sont les conducteurs de travaux et les chefs de chantier qui sont chargés de les faire respecter. Or ils incarnent égal ement, et dans le même temps, l'exigence de cadence qui n'a fait que croître au cours des trente dernières années. Un conducteur de travaux exprime ainsi les contradictions dont il est porteur :

\begin{abstract}
«Les responsables de la sécurité, c'est mon chef, c'est moi, enfin c'est les conducteurs de travaux, ou éventuellement le chef de chantier. C'est pas forcément les mêmes personnes qui sont responsables et qui s'en occupent, en fait. [...] C'est quelque chose qui est contradictoire à la base, presque. C'est une espèce de contradiction entre planning, respecter le planning, et respecter la sécurité, c'est difficile à concilier. [...] On n'arrive pas forcément à vendre [au client] : “Je peux le faire en 18 mois, mais pour le faire vraiment en sécurité, il me faudrait 20 mois", il va te dire: “Faites-le en 18 mois." »
\end{abstract}

Double contradiction, donc: entre la cadence et la sécurité, dont la responsabilité incombe aux mêmes individus; et entre les personnes «qui sont responsables » de la sécurité et celles «qui s'en occupent », de façon distincte. L'appropriation officielle de la gestion de la sécurité par les directions renverse le rapport salarial tel qu'il était théoriquement établi par la loi de 1898: ce ne sont pas les ouvriers qui reprochent aux directions le danger qu'elles sécrètent par leurs directives, ce sont les directions qui reprochent aux ouvriers leur manque de vigilance. Que les directions de chantier fassent prendre des risques aux ouvriers devient officiellement impossible, puisqu'elles sont gardiennes de la sécurité

Un chantier de construction neuve, en hiver. Lorsque les ouvriers arrivent le vendredi matin, le ciel est dégagé mais l'ensemble du chantier est
16. Christophe Dejours, Travail usure mentale, Paris, Bayard, 1993 (1980), p. 87-88.

17. Ibid., p. 89 18. Mais elles ne sont pas entièrement
absentes, et un respect zélé des règles de sécurité peut attirer des moqueries, sinon des réprimandes.
19. Ibid., p. 92. Voir aussi Alain Morice, «Des objectifs de production de connaissances aux orientations méthodologiques: une controverse entre anthropologie et psychodynamique du travail », Revue internationale de psychologie, vol. III, 5 , p. $143-160$ 
recouvert de verglas. La direction du chantier veut faire travailler les ouvriers, qui refusent. Commence alors un mouvement de grève sauvage. Un délégué du personnel fait le lien entre les délibérations des ouvriers et la direction de chantier. Au réfectoire, où s'est improvisée une assemblée générale, le délégué rapporte avoir reproché au chef de chantier qu'il préférait «respecter le planning plutôt que la sécurité». Le chef aurait répondu: «Vous commencez à me faire chier avec la sécurité! ». II aurait ajouté que la prime de productivité sera supprimée ce mois-ci car «les ouvriers nefont pas d'effort». Du côté des ouvriers, il est décidé que si la journée n'est pas déclarée en intempéries, alors il y aura grève le lundi. Le directeur du chantier prévient en public que la journée ne sera pas comptée en intempéries. En réponse, un ultimatum est fixé à l'initiative d'un des ouvriers: «À 9 h 30 on part. S'il se décide pas, c'est nous qui décidons lundi ! ». À $9 \mathrm{~h} 30$, exécution. Le directeur du chantier vient faire une petite harangue avant le départ des ouvriers. «Messieurs, vous êtes des grands garçons, je vais pas vous retenir. Mais aujourd'hui on pouvait travailler. Vous faites du tort à la boîte. - Notre priorité c'est la sécurité, pas le planning, répond le délégué. Les profits de A [I'entreprise générale], c'est pas notre priorité. - Arrêtez, je suis le premier concerné par la sécurité! Je ne vous ferais pas prendre de risques inutiles. Mais aujourd'hui il fait beau, en prenant les mesures de sécurité il était possible de travailler. » Le travail a finalement repris normalement le lundi, la direction du chantier ayant classé la journée chômée en intempéries.

Ce récit d'une prise en charge autonome de leur sécurité par les salariés laisse voir les exigences contraires de productivité portées par les directions, en même temps que leur revendication du monopole de la gestion de la sécurité ( «Arrêtez, je suis le premier concerné par la sécurité»). Mais le caractère exceptionnel de ce genre d'événements rappelle qu'en temps normal la contradiction entre cadence et sécurité reste souterraine, non dite. Au niveau des ouvriers, I'impossibilité de respecter en même temps la cadence et la sécurité, lorsqu'elle n'est pas formalisée politiquement dans ce qui devient, de fait, une contestation du pouvoir des directions, demeure assumée clandestinement. Aussi, une partie du savoir-faire des ouvriers consiste à déterminer où, quand et comment ne pas respecter les règles de sécurité afin de respecter les exigences de cadence

Par exemple, lors de mon premier jour sur un chantier comme aide intérimaire, Patrick, le jeune ouvrier qualifié avec qui I'on m'a placé, se fait très pédagogue. Nous décoffrons plusieurs planchers coulés la veille. Lorsqu'on décoffre le premier plancher, Patrick me fait allonger par terre les étais qu'on a enlevés: «C'est comme ça la sécurité» (debout, ils risquent de tomber et blesser quelqu'un). Au deuxième plancher, changement de tactique, il me les fait mettre debout contre le mur: «Jeteles ai fait mettre par terre la première fois pour que tu saches pour la sécurité, mais maintenant on met contre le mur. Sinon, tu te fatigues à chaque fois à les prendre. Faut que tu t'économises. Si tu suis tous les règlements, t'avances pas».

Difficile de dire, dans ces conditions, que les règles de sécurité «ne rentrent pas» dans la tête des ouvriers; «elles rentrent » suffisamment en tout cas pour qu'ils aient conscience de les enfreindre.

\section{Ouvriers coupables, mais pas responsables}

Dépossédés des modes opératoires, dépossédés de l'organisation de la sécurité: on pourrait croire que les ouvriers ne font qu'obéir aux consignes. Mais s'ils le faisaient, ils ne pourraient pas satisfaire cette exigence fondamentale qu'est la cadence. Alors les ouvriers inventent, improvisent, détournent, créent des modes opératoires informels et potentiellement dangereux. En dépit de l'appropriation officielle de la sécurité par les directions, les ouvriers continuent, par nécessité, de prendre une part dans la gestion de la sécurité, mais «clandestinement et dans l'irrégularitézo ». Dans la mesure où les directions prétendent savoir, pour les ouvriers et à leur place, ce qui est bon pour leur sécurité, les mesures que prennent les ouvriers afin de protéger leur intégrité corporelle doivent être clandestines lorsqu'elles sont en contradiction avec les règles des directions.

Sur le même chantier et avec le même ouvrier, Patrick, je travaille à coffrer des planchers. Une équipe de cadres employés par $\mathrm{B}$ (dont un directeur de la conception du matériel) ainsi que des cadres d'entreprises concurrentes viennent un jour nous observer dans notretravail pour voir le déroulement d'un décoffrage. Patrick et moi décoffrons, sans parler et à toute vitesse; sans nous concerter, nous pensons à desserrer les étais sans taper dessus avec le marteau (comme nous avons coutume de le faire), mais en utilisant le marteau comme levier. Ce qui nous vaut les compliments du directeur de la conception du matériel : «Je voulais vous faire mes félicitations, parce que j'ai vu que vous ne tapiez pas sur les étais. Vous respectez le matériel, et vous respectez vos oreilles. C'est important de travailler en sécurité, c'est bien ». Patrick et moi échangeons un sourire complice Car, comme nous le faisions hier, nous recommencerons demain à taper sur les étais: tant pis pour nos oreilles, mais 


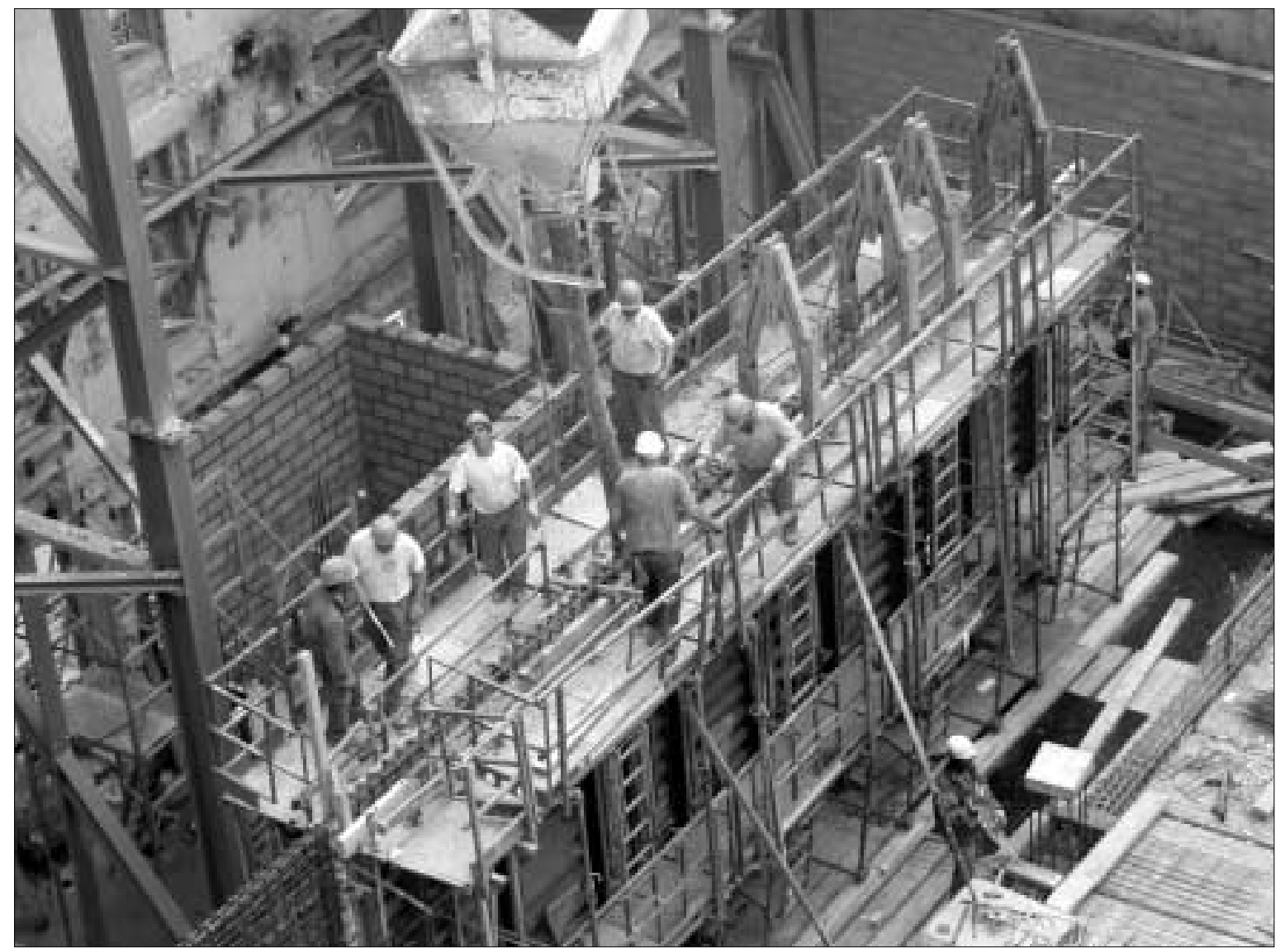

COULER LE BÉTON, un moment tendu. 
nous savons que faire levier avec le marteau est trop mauvais pour le dos. Une fois les cadres partis, c'est Patrick qui rompt le silence: « Les félicitations, c'est mon salaire. J'en ai rien à foutre de ses félicitations».

Néanmoins, cette dépossession de la sécurité «officielle» contribue à un sentiment de dépossession des objectifs mêmes de la sécurité La bonne exécution de I'ouvrage, la protection de son intégrité corporelle ou de celle des autres semblent en situation, dans les échanges entre ouvriers, éclipsées au profit du seul rapport à la hiérarchie: «Si le type de la sécu me voit, je dégage tout de suite»; «Descends! II y a le type de la sécurité»; «Si le conducteur de travaux voit tes gars comme ça, je vais me faire allumer » : voilà letype d'injonctions au respect des règles de sécurité qui circulent sur les chantiers. «Je vais me faire al lumer » est l'argument roi, une sorte d'équivalent général des justifications, le seul que tous peuvent invoquer et entendre. La plus solide des justifications du respect de règles de sécurité tient dans cette peur affichée de la réprimande hiérarchique, non dans la nécessité de préserver la sécurité. II n'y a pas là d'inconscience des ouvriers, mais au contraire une conscience aiguë des rapports hiérarchiques dans lesquels ils sont pris. Si I'anticipation du reproche hiérarchique est l'argument suprêmement légitime, l'argument qui met tout le monde d'accord, c'est là un résultat de la division entre concepteurs et exécutants: dépossédés de la conception, de la direction, de l'organisation du travail et en particulier de la sécurité, les exécutants ont en commun de faire leur travail d'abord et avant tout pour satisfaire les consignes de leurs supérieurs.

«La sécurité, c'est de la responsabilité de tous», proclament volontiers les promoteurs et prescripteurs de la sécurité Mais le terme ambigu de «responsabilité» masque que la responsabilité dont sont investis les cadres en matière de sécurité est del'ordre du pouvoir, tandis que celle des ouvriers est de l'ordre de la culpabilité: coupables mais pas responsables. Aux uns la charge de surveiller que la sécurité est respectée, aux autres celle de rendre compte de leurs actions, à l'occasion jugées «irresponsables». Entre les deux: le «réel du travail 21 », organisation, matériel, cadence, qui empêche concrètement les ouvriers de respecter les règles de sécurité prescrites. Quand survient I'accident, la direction se doit de chercher des coupables. Il y en aura forcément, comme l'explique un chef d'équipe intérimaire, à propos d'un accident récent qui a fait deux morts parmi les ouvriers sur un chantier où il travaillait: «lls sont toujours à chercher un coupable, il faut qu'ils en trouvent un. Mais tu pousses, tu pousses à aller plus vite, c'est ça le truc, les mecs ils ont serré trop vite et voilà... ». Quelques semaines après m'avoir confié ce sentiment, ce chef d'équipe régulièrement pressé par ses supérieurs, souvent stressé, effectuant de nombreuses heures supplémentai res, se cassait la jambe en essayant d'installer, seul, un coffrage de poteau descendu par la grue. Un manœuvre du même chantier commentait l'accident dans des termes proches de ceux du chef d'équipe: «C'est toujours comme ça, ils ont dit que c'était de sa faute. Alors que c'est eux qui poussent. Ils crient sur toi, “je veux que le travail avance". Et quand ils arrivent, ils disent que c'est ta faute». Le geste accidentel ou meurtrier sera toujours, nécessairement, celui d'un exécutant; ce sera toujours un exécutant qui n'aura pas bien serré, fixé ou posé telle sécurité C'est en mettant en procès ces gestes au cas par cas, comme autant d'infractions particulières, isolées, que la direction de chantier passe sous silence le cadre organisationnel de ces gestes et procède à son propre acquittement.

Aussi, tout incite les ouvriers à devenir de plus en plus «irresponsables » et notamment à masquer leurs erreurs, à fuir les responsabilités, à éviter les zones critiques pour ne pas être critiqués, quand bien même leur intervention serait utile.

\begin{abstract}
Par exemple, sur un chantier de rénovation, je suis manœuvre intérimaire auprès d'un ouvrier qualifié, Fabrice. Alors que j'entre avec lui dans une pièce où des vitres sont déjà posées aux fenêtres, un courant d'air fait se refermer violemment la fenêtre laissée ouverte. Le carreau se brise, une partie tombe à l'extérieur (côté rue), on a l'impression d'entendre un cri. Je veux aller voir, Fabrice me dit: «Ça va pas, non? Allez viens, on se casse. Si on te demande, tu dis que t'as rien vu ». Quelques minutes plus tard, des chefs montent et nous demandent si on a vu la vitre tomber. Réponse de Fabrice: «Ouais, on a entendu un grand blam, on était dans la pièce à côté... - Non, mais on sait bien que c'est pas votre faute, mais il faut prévenir lechef. A près le client, il sait, ça fait moyen ». II n'y a pas de blessé, mais le pare brise d'un taxi garé en bas est détruit.
\end{abstract}

\section{Déresponsabilisation en cascade: la sécurité à l'épreuve de l'externalisation}

En séparant grossièrement concepteurs et exécutants, cadres et ouvriers, il s'agissait ici de souligner la frontière la plus significative. Mais c'est une frontière qui se décline à l'intérieur du collectif ouvrier luimême: la dynamique qui, dans un même mouvement, insécurise du fait des conditions de travail, et 
déresponsabilise/culpabilise au niveau de la gestion de la sécurité, se reproduit de manière d'autant plus forte que les travailleurs appartiennent aux périphé ries du collectif.

Les prix bas auxquels doivent traiter les entreprises sous-traitantes les poussent à sacrifier la sécurité à la vitesse d'exécution, davantage encore que l'entreprise générale. Pour les intérimaires (et donc a fortiori pour les intérimaires de sous-traitants), la précarité de l'emploi incite à prendre des risques qu'ils éviteraient autrement: se trouvent mis en balance un risque contre un autre, le risque corporel contre le risque de perdre son emploi, ce dernier étant accru par l'intérim²2. Quant aux sans-papiers, assez nombreux notamment parmi les ferrailleurs et les manœuvres, à supposer que leurs employeurs soient disposés à veiller à leur sécurité, l'irrégularité de leur séjour leur fait craindre toute déclaration officielle, même d'accident de travail.

Spécifiquement insécurisés, les travailleurs intérimaires et/ ou sous-traitants sont égal ement spécifiquement déresponsabilisés. Par exemple, si l'on considère la distribution des postes de secouristes: sur les chantiers observés, ceux qui avaient lestatut de secouriste étaient des conducteurs de travaux de l'entreprise générale, des chefs de chantier et d'équi pe de l'entreprise générale ou d'une entreprise sous-traitante, et plus rarement des ouvriers qualifiés embauchés par l'entreprise générale. Les seuls à être exclus systématiquement de la fonction de secouristes sont les exé cutants des entreprises sous-traitantes et les intérimaires. Cela s'explique en partie par le fait qu'ils sont les éléments les moins stables du chantier, alors que l'entreprise générale doit justifier en permanence auprès del'inspection du travail d'une proportion minimale de secouristes. Mais en réalité, un certain nombre d'exécutants sous-traitants et intérimaires, fidélisés par I'entreprise ou par le chef de chantier, ne sont pas moins stables que les autres. II est donc question de politique, pas de technique, dans l'attribution des postes de secouristes (ce que confirme par ailleurs la sous-représentation des ouvriers embauchés de l'entreprise générale, hors chefs d'équipe). Pourtant, ceux qui ont le plus de chances d'avoir un accident sont les ouvriers; et la personne la plus proche d'un ouvrier, dans ces cas-là, est presque toujours un autre ouvrier. Aussi, la formation des ouvriers au secourisme, et des ouvriers davantage que des cadres, trouverait sa justification sur le plan strict de la sécurité. Mais la gestion taylorienne de la sécurité en chantier s'en accommoderait difficilement; ce serait une brèche dans le pouvoir de la hiérarchie si son autorité était écartée précisément dans les situations les plus extrêmes. Au regard de la sécurité, la présence de sous-traitants et d'intérimaires, composant la majorité des effectifs, a deux conséquences. D'une part, celle d'un éclatement du collectif ouvrier, et un éclatement hiérarchisé: les sous-traitants et les intérimaires ont généralement moins de droits que les ouvriers de l'entreprise géné rale, et moins de possibilités de les faire valoir. Ce qui fragilise une éventuelle coalition visant à prendre en charge collectivement la sécurité

\begin{abstract}
Dans le cas du mouvement de grève spontané relaté plus haut, la partition était claire: le mouve ment était mené par les ouvriers embauchés de l'entreprise générale, et quelques-uns des intérimaires de l'entreprise générale. La majorité des intérimaires del'entreprise générale, ainsi queles ouvriers (embauchés et intérimaires) des soustraitants restaient en retrait, soit en approuvant le mouvement mais en demeurant en marge de l'assemblée qui en décidait, soit en le critiquant: «Nous les intérimaires, on va être niqués»; «Leur délégué c'est pas pour nous, on n'a rien à voir avec A. Si les A [les ouvriers embauchés de A] travaillent pas, ils seront payés, mais nous on va être niqués». Prédiction en partie réaliste: du côté des intérimaires, chacun a dû se débrouiller avec son agence d'intérim pour se voir payer ses indemnités d'intempéries, et plusieurs agences, comme souvent, ont manqué à cette obligation. Quinze jours plus tard, quelques coffreurs intérimaires ont fait une grève, demeurée isolée (en dépit des proclamations de solidarité «jusqu'au bout » des embauchés lors de la première grève), pour réclamer le paiement de leurs indemnités. Ils ont gagné, mais les ferrailleurs et manœuvres intérimaires qui se trouvaient dans le même cas ne se sont pas mobilisés.
\end{abstract}

D'autre part, au quotidien, les ouvriers de l'entreprise générale occupent vis-à-vis des sous-traitants et des intérimaires une position homologue à celle des cadres vis-à-vis des ouvriers dans leur ensemble: celle de gardiens de la sécurité face à des exécutants potentielle ment irresponsables. Cette réputation d'irresponsabilité est en partie justifiée par le fait que les travailleurs sous-traitants ou intérimaires, plus précaires et plus contraints par les exigences de cadence, ont tendance à prendre plus de risques et à être moins regardants sur la sécurité. Mais veulent-ils prendre soin de la sécurité, qu'on ne les laisse pas toujours faire, puisqu'ils n'en sont pas les responsables officiels. Les outils de sécurité (garde-corps par exemple) sont, comme tous les outils collectifs du chantier (notamment la grue), achetés ou loués par l'entreprise générale, et ce sont 
ses ouvriers propres qui ont le monopole de leur usage légitime. Aussi un sous-traitant qui veut prendre en charge la sécurités'exposet-il à être renvoyé à ses attributions: la production et rien d'autre.

Non seulement ceux qui conçoivent la sécurité ne sont pas ceux qui la font; mais ce ne sont pas non plus ceux qui sont supposés en bénéficier. Ce décalage a ses racines dans la constitution même du travail salarié. Il a cependant des formes et des conséquences spécifiques dans le cadre du travail en chantier. Mettre en lumière la taylorisation de la sécurité revient à extraire la question de la sécurité du domaine de la technique où elle est souvent confinée, et à décrire la confi- guration sociale de prise en charge de la sécurité qui prévaut sur les chantiers: cette prise en charge se mue en une avalanche de prescriptions adressées aux ouvriers, constamment soupçonnés d'y déroger. Pris entre les exigences de cadence et les prescriptions de sécurité, les ouvriers n'ont plus le choix que de prendre des risques, et de cacher qu'ils prennent des risques. La réglementation de l'entreprise en matière de sécurité n'a donc pas pour unique effet la protection de l'intégrité physique des ouvriers. À la limite, elle est même susceptible d'avoir l'effet inverse: sans éliminer les conduites à risque, elle impose le silence à leur sujet, et fait ainsi obstacle aux tentatives de reprise en main de leur sécurité par les salariés. 